Introduction There is some controversy in the literature regarding the outcomes of pregnancies complicated by antepartum bleeding of unknown origin (ABUO).

Objective To explore the risk of adverse maternal and perinatal outcomes in women with ABUO occurring after the first trimester of pregnancy.

Methods Cohort study based on data extracted from the Aberdeen Maternity and Neonatal Databank. The study population was all primigravidae delivering in Aberdeen Maternity Hospital between 1976 and 2010. Exposure was antepartum haemorrhage occurring after the first trimester not attributable to placenta praevia or abruption. Data were analysed using univariate and multivariate statistical methods

Results Between 1976 and 2010, there were 7,517 women with ABUO and 68,423 women without. Women with ABUO were more likely to be smokers, belong to lower social class and have slightly higher body mass index. Multivariate analysis revealed that non-specific APH was a significant risk factor for induced labour $(\mathrm{OR}=1.23,95 \% \mathrm{CI}=1.16,1.31)$, preterm delivery $(\mathrm{OR}=2.30,95 \% \mathrm{CI}=2.11,2.50)$, postpartum haemorrhage $(\mathrm{OR}=1.15,95 \% \mathrm{CI}=1.06,1.25)$, Apgar score less than 7 at 1 minute $(\mathrm{OR}=1.12,95 \% \mathrm{CI}=1.05,1.21)$, and at 5 minutes $(\mathrm{OR}=1.25,95 \% \mathrm{CI}=1.04,1.50)$. There was no significant association detected with preeclampsia, mode of delivery or perinatal death.

Conclusion Pregnancies complicated by ABUO are at greater risk of delivery related and neonatal adverse outcomes attributable to preterm birth, some of which is iatrogenic.

\section{PP.23 MEASUREMENTS OF AMNIOTIC FLUID: ASSOCIATION AND PREDICTION OF SMALL FOR GESTATIONAL AGE AND COMPROMISE OF FETAL WELLBEING: A SYSTEMATIC REVIEW AND META-ANALYSIS}

doi:10.1136/archdischild-2013-303966.303

${ }^{1,2} \mathrm{RK}$ Morris, ${ }^{2,3} \mathrm{CH}$ Meller, ${ }^{1,4} \mathrm{JA}$ Tamblyn, ${ }^{5} \mathrm{GM}$ Malin, ${ }^{8} \mathrm{RD}$ Riley, ${ }^{1,2,8} \mathrm{MD}$ Kilby, ${ }^{6} \mathrm{SC}$ Robson, ${ }^{7}$ KS Khan. 'Division of Reproduction, Genes and Development, University of Birmingham, Birmingham, UK; ${ }^{2}$ Fetal Medicine Centre, Birmingham Women's Hospital, Birmingham, UK; ${ }^{3}$ Obstetrics Division, Hospital Italiano de Buenos Aires, Buenos Aires, Argentina; ${ }^{4}$ University Hospital North Staffordshire, Stoke-On-Trent, UK; ${ }^{5}$ School of Clinical Sciences, University of Nottingham, Nottingham, UK; ${ }^{6}$ Institute of Cellular Medicine, Newcastle University, Newcastle, UK; ' Women's Health Research Unit, The Blizard Institute, Barts and The London School of Medicine, Queen Mary, University 0, London, UK; ${ }^{8}$ School of Health and Population Sciences, University of Birmingham, Birmingham, UK

Objective Evaluate the strength of association and predictive value of measurements of amniotic fluid volume (AFV) for small for gestational age and compromise of fetal wellbeing.

Design Systematic literature review with random effects meta-analysis to compute summary odds ratios (OR) to assess prognostic association and assess predictive ability with sensitivity, specificity and likelihood ratios. Study characteristics, design, methodological and reporting quality were objectively assessed.

Data Sources and Eligibility Systematic search (inception to October 2011) for studies comparing AFV measures and outcomes of fetal size or wellbeing.

Results 43 studies reporting 244,493 fetuses included. Strong associations between oligohydramnios (heterogenic definition) and birth weight $<10^{\text {th }}$ centile [OR $6.31(4.15-9.58)$ ] in a high risk population (6 studies, 28510 fetuses), and mortality [Neonatal death OR 8.72 (2.43-31.26) 6 studies, 55735 fetuses, and perinatal mortality in a high risk population OR 11.54 (4.05-32.9) 2 studies, 27891 fetuses] were identified. There was no significant association between oligohydramnios and abnormal cord $\mathrm{pH}$ or adverse perinatal outcome.
There was no significant association between polyhydramnios (heterogenic definitions) and poor fetal growth [Birth weight $<10^{\text {th }}$ centile OR 0.37 (0.07-1.95)]. A strong association between polyhydramnios (maximum pool depth $>8 \mathrm{~cm}$ or amniotic fluid index $\geq 25 \mathrm{~cm}$ ) and birth weight $>90^{\text {th }}$ centile [OR 11.41 (7.09-18.36) 1 study, 3960 fetuses] was found. Despite strong associations predictive ability was poor with significant heterogeneity despite subgroup analysis.

Conclusion Oligohydramnios is associated with poor fetal growth and mortality. Polyhydramnios is associated with BW $>90^{\text {th }}$ centile. Despite strong associations, overall predictive ability was poor. MPD accuracy was slightly improved over AFI and is the recommended technique.

\section{PP.24 PLACENTAL VOLUME CAN BE ACCURATELY MEASURED USING TWO- AND THREE-DIMENSIONAL ULTRASOUND NEAR TERM}

doi:10.1136/archdischild-2013-303966.304

1,2LE Higgins, ${ }^{1} \mathrm{CP}$ Sibley, ${ }^{1,2} \mathrm{AEP}$ Heazell, 1,2ED Johnstone. 'Maternal and Fetal Health Research Centre, University of Manchester, Manchester, UK; ${ }^{2}$ St. Mary's Hospital, Central Manchester University Hospitals NHS Trust, Manchester, UK

Introduction Stillbirth and fetal growth restriction are associated with lower placental weights compared to live, appropriately grown infants. Sonographic measures of placental size have been used to predict later pregnancy complications but have not been related back to true placental size. We aimed to validate measures of placental size in the third trimester of pregnancy.

Methods Ultrasonographic placental examination was performed in 30 singleton pregnancies of 35-42 weeks' gestation within seven days of delivery. Placental length, width and depth were measured using two-dimensional (2D) ultrasound, placental volume was estimated by a novel elliptical model, Kliman placental gas gauge and two three-dimensional (3D) ultrasound techniques (rotational (VOCAL) and slicing methods). Following delivery, the placenta was measured, weighed and volume determined. Sonographic and true placental measures were compared using linear regression.

Results Elliptical (but not Kliman placental gas gauge) 2D and all $3 \mathrm{D}$ ultrasound estimates of placental volume each related significantly with true placental volume with similar accuracy (Table 1) but not to placental weight $(p>0.05)$. VOCAL demonstrated higher accuracy than the elliptical model at the smallest placental volumes.

\section{Abstract PP.24 Table 1}

\begin{tabular}{llll}
\hline & & $\mathbf{r}^{2}$ & $\mathbf{p}$ \\
\hline Ellipse & & 0.27 & 0.0033 \\
Kliman placental gas gauge & 0.054 & 0.21 \\
VOCAL & $30^{\circ}$ & 0.31 & 0.0018 \\
& $15^{\circ}$ & 0.67 & 0.0042 \\
Slicing & $10 \mathrm{~mm}$ & 0.24 & 0.0064 \\
& $5 \mathrm{~mm}$ & 0.29 & 0.0025 \\
\hline
\end{tabular}

Conclusion True placental volume can be accurately predicted using $2 \mathrm{D}$ and $3 \mathrm{D}$ ultrasound in the third trimester. 3D ultrasound may improve detection of the abnormally small placenta. Further research is required to establish whether this measurement can predict poor pregnancy outcomes related to placental disease.

\section{PP.25 INTERPREGNANCY CHANGES IN MATERNAL WEIGHT AND BODY MASS INDEX}

doi:10.1136/archdischild-2013-303966.305

DA Crosby, M Collins, V O'Dwyer, A O'Higgins, N Farah, MJ Turner. Coombe Women and Infant's University Hospital, Dublin, Ireland 
Objective This longitudinal study examined changes in maternal weight and Body Mass Index (BMI) in the early pregnancy between a woman's first and second baby.

Methods We studied women more than 18 years old with a singleton pregnancy who delivered their first baby weighing $\geq 500$ grammes in 2009 and who re-attended for antenatal care with a subsequent ongoing pregnancy before January $1^{\text {st }} 2012$. Maternal weight and height were measured accurately before 18 weeks gestation in both pregnancies and BMI was calculated.

Results Of the 3284 primigravidas, the mean weight at the first antenatal visit was $66.4 \mathrm{~kg}$ (SD 12.7). The mean BMI was $24.5 \mathrm{~kg} / \mathrm{m}^{2}$ (SD 4.6), and $11.3 \%(\mathrm{n}=370)$ were obese. Of these 3284 women, $1220(37.1 \%)$ re-attended for antenatal care before 2012 after confirmation of an ongoing pregnancy. Of the 1220 women who re-attended, $788(64.6 \%)$ had gained weight (mean $4.6 \mathrm{~kg}$ SD 3.9), 402(33.0\%) had lost weight (mean $3.0 \mathrm{kgs} \mathrm{SD} 2.9$ ) and 30 (2.4\%) had maintained their weight. As a result, $20.2 \%(\mathrm{n}=247)$ were now in a higher BMI category and $4.8 \%(n=58)$ had become obese; $5.8 \%(n=71)$ were in a lower BMI category and $1.2 \%(n=15)$ were no longer obese. These early pregnancy weight changes were influenced by maternal age, but not by the duration of the interpregnancy interval.

Conclusion As two thirds of women gain weight in the shortterm after delivery of their first baby, we recommend that the advice women get before and during pregnancy needs to be reinforced postpartum.

\section{PP.26 SUSTAINABLE VOLUNTEERING AND ITS EFFECT ON UGANDAN STILLBIRTH RATES}

doi:10.1136/archdischild-2013-303966.306

'K MacLeod, ' 2 L Ackers. 'Mersey Deanery, Liverpool, UK; ${ }^{2}$ University of Liverpool, Liverpool, UK

Background The Ugandan Maternal and Newborn Hub is a THET funded initiative focused on implementing Sustainable Volunteering Projects (SVPs) to improve maternal and newborn health. Annually, there are 2.65 million stillbirths worldwide and almost $90 \%$ occur in low-resource settings. Up to $70 \%$ of stillbirths occur in the intrapartum period; frequently these are associated with suboptimal care.

Methods Through a SVP, from January to June 2012, we undertook a quality improvement project aiming to reduce intrapartum stillbirths at Mbarara Hospital in Uganda. Our quality improvements included regular audit presentations, implementation of a labour ward board and skills and drills training. We classified suboptimal care into types of delay using Thaddeus \& Maine Three Delay Model.

Results In this 6 months period, there were 102 intrapartum stillbirths. Most stillbirths were singleton pregnancies (99\%) with a mean maternal age of 24 years and a mean parity of $2.34 \%$ of women were unbooked. Suboptimal factors contributing to

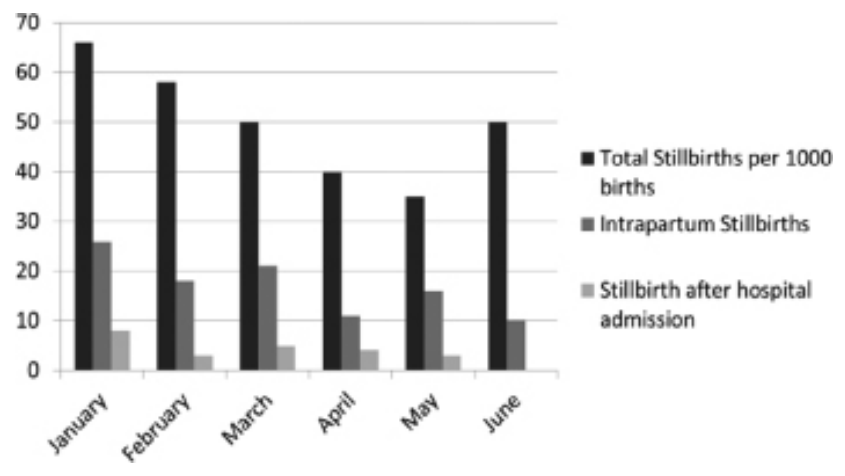

Abstract PP.26 Table Stillbirths Figures Mbarara Hospital 2012 intrapartum stillbirths were identified in $86.4 \%$ of cases, $22 \%$ were related to care whilst admitted. Intrapartum stillbirths occurring after hospital admission reduced after introduction of quality improvement measures: see table.

Discussion Basic and comprehensive emergency obstetric care is an important intervention to reduce intrapartum stillbirths in the developing world. SVPs are an effective way of individualising quality improvement measures and improving outcomes.

\section{PP.27 MICRO-VASCULAR DISEASE AT BOOKING IN T1DM AND ASSOCIATED RISK OF DEVELOPING PRE-ECLAMPSIA}

doi:10.1136/archdischild-2013-303966.307

${ }^{1} \mathrm{~N}$ Patel, ${ }^{2} \mathrm{~A}$ Brackenridge, ${ }^{3} \mathrm{~A}$ Kanji, ${ }^{1} \mathrm{D}$ Pasupathy, ${ }^{4} \mathrm{D}$ Rajasingam. 'Division of Women's Health, Women's Health Academic Centre, King's College London, King's Health Partners, London, UK; 'Department of Diabetes, Guy's and St Thomas' NHS Foundation Trust, London, UK; ${ }^{3}$ School of Medicine, King's College London, London, UK; ${ }^{4}$ Division of Women's Services, Guys and St Thomas' NHS Foundation TRust, London, UK

Background and Aims Micro-vascular disease affects the majority of T1DM patients and is directly related to both duration and glycemic control of diabetes. The aim of this study is to identify the association of micro-vascular disease at booking and subsequent risk of developing pre-eclampsia during pregnancy.

Methods Retrospective cohort study of women with T1DM $(\mathrm{n}=37)$ on continuous subcutaneous insulin infusions booked at Guy's \& St Thomas' Hospital between November 2010 and April 2012. Micro-vascular disease at booking was, defined as nephropathy or/and retinopathy at booking.

Results The prevalence of micro-vascular disease at booking was $54.1 \%(n=20)$. No significant difference were seen in age (33.5 years vs 35.0 years; $p=0.28)$, HbA1c (7.5 vs $6.9 ; p=0.11)$, length of diabetes $(16.0$ years versus 23.0 years; $p=0.14)$ compared to women without micro-vascular disease.

Presence of micro-vascular disease was significantly associated with the pre-eclampsia $(55.0 \%$ vs $17.6 \%$; $\mathrm{p}=0.02$; unadjusted $\mathrm{OR}$ 5.70; CI 1.24-26.26). This association was not explained by maternal age, duration or control of diabetes, parity, BMI and maternal hypertension (adjusted OR 4.92; CI 0.7-32.3).

The rate of LGA and SGA was $51.3 \%$ and $5.4 \%$ respectively. Women with micro-vascular disease had higher rates of SGA $(10.0 \%$ vs $0.0 \%$; $p<0.05)$ and LGA $(65.0 \%$ vs $35.0 \%$; $<<0.05)$.

\section{PP.28 CERVICAL CERCLAGE: ARE ALL TECHNIQUES SUCCESSFUL?}

doi:10.1136/archdischild-2013-303966.308

${ }^{1}$ EA Bonney, ${ }^{2} \mathrm{~A}$ Atkinson, ${ }^{2} \mathrm{R}$ Lancaster, ${ }^{1} \mathrm{JJ}$ Walker, ${ }^{1} \mathrm{NAB}$ Simpson. 'Division of Clinical Sciences, Leeds Institute of Molecular Medicine, University of Leeds, Leeds, UK; ${ }^{2}$ School of Medicine, University of Leeds, Leeds, UK

Objective Preterm birth is a global public health issue. In women considered high risk, insertion of a cervical cerclage has been shown to reduce this risk. We present findings from a retrospective cohort evaluating the success of different cerclage procedures (Shirodkar, McDonald and Transabdominal) in a tertiary level obstetric unit.

Study design Retrospective data was collected for 200 women who underwent a cerclage procedure at Leeds Teaching Hospitals NHS Trust between August 2000 and October 2010. Exclusion criteria for the study included multiple pregnancy, insertion of more than one cerclage in a single pregnancy, or an incomplete data record. Success was measured by delivery of a live baby $\geq 34$ weeks. Mean gestational age (MGA) for each group was also calculated. Statistical analysis was performed using Fisher's exact test. 